\title{
A Novel Strategy for Proficient Channel Limitation Equalizer
}

\author{
P.LAKSHMI NAGA SINDHU, M.tech student \\ D.S.SAMBASIVA RAO, HOD
}

Department of Electronics and Communication Engineering

Prasiddha Collage of Engineering and Technology

Anathavaram, India.

\begin{abstract}
A time-domain equalizer (TEQ) employed in cascade with the channel yields an actual impulse response that is squatter than the channel impulse response. Time-domain equalization is critical in reducing channel state dimension in maximum likelihood sequence approximation, and inter-carrier and inter-symbol interference in multicarrier systems. This project evaluates two TEQ design methods docile to cost-effective real-time implementation: least mean squared error (LMSE) and extreme shortening SNR (ESSNR) methods. We condense the complexity of computing the matrices in the ESSNR and LMSE designs by a factor of 140 and a factor of 16 (respectively) relative to existing approaches, without degrading performance. We prove that an infinite length ESSNR TEQ with unit norm TEQ constraint is symmetric. A symmetric TEQ halves FIR implementation density, enables parallel training of the frequency-domain equalizer and TEQ, reduces TEQ training complexity by a factor of 4 and doubles the length of the TEQ that can be designed using fixed-point arithmetic, with only a small loss in bit rate. Simulations are presented for designs with a symmetric TEQ or target impulse response.
\end{abstract}

Keywords:TEQ, LMSE, ESSNR.

\section{INTRODUCTION}

Signal processing and communications have been complementary fields of electrical engineering for a long time. Although most of the basic processing tools utilized in the design of communication systems clearly come from the signal processing discipline, e.g., Fourier transform and modulation schemes, others are specifically designed for communication purposes, such as information theory and error-correcting codes. In turn, signal processing experts have also been influenced by this cross-fertilization and expanded their research activities into various communication applications.

This mutual influence and interaction, however, has not been as strong in the area of discrete-time multi-rate signal processing.

Highlighting the fundamentals of orthogonal sub-band transforms from a time-frequency perspective, this project illustrates how both disciplines would benefit from a stronger cooperation on this topic. Several popular communication applications can be described in terms of synthesis/analysis configuration (transmultiplexer) of sub-band transforms. Code division multiple access (CDMA), frequency division multiple access (FDMA), and time division multiple access (TDMA) communication schemes can be viewed from this perspective. In particular, FDMA [which is also called orthogonal frequency division multiplexing (OFDM)] or discrete multi-tone (DMT) modulation-based systems have been more widely used than the others.

The orthogonality of multicarriers was recognized early on as the proper way to pack more subchannels into the same channel spectrum [20], [35], [36], [68]. This approach is meritful particularly for the communication scenarios where the channel's power spectrum is unevenly distributed. The sub-channels (subcarriers) with better power levels are treated more favourably than the others. Therefore, this approach provides a vehicle for an optimal loading of sub-channels where channel dynamics are significant. The subcarrier orthogonalityrequirements were contained in a single domain in conventional communication schemes. Namely, they are the orthogonality in frequency (no interference between different carriers or subchannels) and the orthogonality in time (no interference between different sub-symbols transmitted on the same carrier at different time slots). If this property is ensured, multichannel communication is achieved naturally.

Originally, the multicarrier modulation technique was proposed by using a bank of analogNyquist filters, which provide a set of continuous-time orthogonal functions. However, the realization of strictly orthogonal analog filters is impossible. Therefore, the initial formulation was reworked into a discrete-time model. The steps of this discrete-time model are summarized as follows. A digital computation first evaluates samples of the continuous signal that is to be transmitted over the channel. Then, these samples drive a digitalto-analog converter (DAC), which generates the actual transmitted signal. This discrete model makes explicit use of a structure that is similar to the orthogonal synthesis/analysis filter bank or transmultiplexer displayed in Fig. 1(b). 
Transmultiplexers were studied in the early 1970's by Bellangeret al. [37] for telephony applications. Their seminal work was one of the first dealing with multi-rate signal processing, which has matured lately in the signal processing field. Since complexity is an important issue in all of these applications, the discrete Fourier transform (DFT) basis is usually chosen as the set of orthogonal subcarriers [31], [38], [66]. In addition, it has been shown that the DFT-based transmultiplexers allow efficient channel equalization, which make them attractive. The orthogonality conditions and implementation of discrete-time (digital) function sets are much easier to use than the ones in the continuous-time domain (analog case) [6], [7]. This is the first point where DSP tools can be useful. In addition, it has been shown in [66] that the only Nyquist filter, which allows the time and frequency orthogonalities when modulated by a DFT, is a rectangular window (time) function. All other modulations using more selective window functions can only approximate the orthogonality conditions. There are some ways to circumvent such restrictions, which are discussed further. On the other hand, more general orthogonality conditions need to be satisfied for other transform bases. This is currently an active research topic in the field.

\section{CAPACITY OF A MULTICARRIER CHANNEL}

Modulation with a N-point IFFT generates two one-dimensional (real) (DC and Nyquist frequencies), and N/2 - 1 two-dimensional (complex) subchannels. For an adequately long cyclic prefix, it is reasonable to assume that the channel gain and noise power in each subchannel is flat. In this case, each subchannel can be modeled as an independent AWGN channel. The capacity of a multicarrier channel can be written in terms of bits per symbol as [2].

$$
C_{D M T}=\sum_{i \epsilon S} \log _{2}\left(1+S N R_{i}^{M F B}\right) \text { bits/symbol }
$$

The maximum achievable bits per symbol, on the other hand, can be written as

$$
b_{D M T}=\sum_{i \in S} \log _{2}\left(1+\frac{S N R_{i}^{M F B}}{\prime}\right) \quad \text { bits/symbol }
$$

where $\mathrm{i}$ is the subchannel index, $\mathrm{S}$ is the set of the indices of the used $\mathrm{N}$ subchannels out of $\mathrm{N} / 2+1$ subchannels, $\mathrm{SNR}_{\mathrm{i}}{ }^{\mathrm{MFB}}$ is the matched filter bound of the SNR in the ithsubchannel and is defined below in (3), and $\square$ is the SNR gap for achieving Shannon channel capacity and is assumed to be constant over all subchannels [21]. The bit rate can be calculated from (2) by multiplying by the symbol rate, which is $4 \mathrm{kHz}$ in the ADSL standards. In downstream G.DMT transmission, for example, the symbol rate is calculated as the sampling frequency (2.208 $\mathrm{MHz})$ divided by samples per symbol $(512+32=544)$ and multiplied by $68 / 69$ to adjust for the fixed symbol in every frame of 69 symbols.

The SNR gap is a function of several factors, including the modulation method, allowable probability of error Pe, gain of any coding applied $\gamma_{\text {eff }}$, and desired system margin $\gamma_{\mathrm{m}}$. The SNR gap can be approximated in the case of quadrature amplitude modulation (QAM) as [21].

$$
'=\frac{\gamma_{\mathrm{m}}}{3 \gamma_{\mathrm{eff}}}\left(\mathrm{Q}^{-1}\left(\frac{\mathrm{P}_{\mathrm{e}}}{2}\right)\right)
$$

Assuming that the input signal and noise are wide sense stationary, the SNR in the ithsubchannel can be defined as

$$
S N R_{i}^{M F B}=\frac{S_{x, i}\left|H_{i}\right|}{S_{n, i}}
$$

where $S_{x, i}$ and $S_{n, i}$ are the transmitted signal and channel noise power, respectively, and $H_{i}$ is the gain of the channel spectrum in the $\mathrm{i}^{\text {th }}$ subchannel. We also assume that the subchannels are narrow enough so that the channel frequency response and transmitted signal power spectrum are constant in each subchannel. The definition in (4) does not include the effect of ISI or any equalizer. It is the maximum achievable SNR or the matched filter bound (MFB). If the channel causes ISI or an equalizer has been used, then the definition has to be modified.

\section{The Geometric TEQ Method:}

Al-Dhahir and Cioffi [11] propose a method to incorporate the optimization of achievable bit rate into the TEQ design. The goal is to use the ultimate performance measure as an objective function in the TEQ design procedure. Their derivation starts with the definition of the GSNR which is a useful measure related to the bit rate 


$$
G S N R='\left(\left[\prod_{i \in S}{ }^{1+S N R}\right]\right)
$$

Maximizing the GSNR is equivalent to maximizing the bit rate [11]. In (5), the subchannel SNR defined in (4) is modified to include the effect of the equalizer [11].

$$
\operatorname{SNR}_{i}^{E Q}=\frac{S_{x, i}\left|B_{i}{ }^{2}\right|}{S_{n, i}\left|W_{i}{ }^{2}\right|}
$$

where $\mathrm{S}_{\mathrm{x}, \mathrm{i}}$ is the signal power, $\mathrm{S}_{\mathrm{n}, \mathrm{i}}$ is the noise power, and $\mathrm{B}_{\mathrm{i}}$ and $\mathrm{W}_{\mathrm{i}}$ are the gain of $\mathrm{b}$ and $w$ in the $\mathrm{i}^{\text {th }}$ subchannel, respectively.

Recall that the equalized channel can be modeled as a delay by $\Delta$ samples followed by an FIR filter whose impulse response is the TIR. Al-Dhahir and Cioffi state the optimum TIR problem

Here, $\mathrm{R} \Delta$ is a channel-dependent matrix and $\mathrm{MSE}_{\max }$ is a channel-dependent parameter which limits the MSE. The nonlinear constrained optimization problem in (6) does not have a closed form solution, but may be solved by numerical methods.

The MGSNR TEQ method is not optimum (in the sense of maximizing bit rate) due to several approximations. One approximation is in the GSNR definition itself| the method maximizes an approximation to the actual GSNR. The objective function is derived based on the assumption that the TIR and the TEQ coefficients are independent. However, this is not the case because the "optimal" TEQ coefficients are calculated from the "optimal" TIR coefficients using

$$
\mathbf{w}_{o}^{T}=\mathbf{b}_{o}^{T} \mathbf{R}_{x y} \mathbf{R}_{y y}^{-1}
$$

Where $w_{o}$ and $b_{o}$ are the "optimal" TEQ and TIR vectors, respectively, and $R_{x y}$ and $R_{y y}$ the input-output crosscorrelation and output autocorrelation, respectively.

The most important approximation, however, is in the definition of the subchannel $\mathrm{SNR}, \mathrm{SNR}^{\mathrm{EQ}}{ }_{\mathrm{i}}$ in (6), which includes the effect of the equalizer but not the effect of the ISI, even though the objective of the TEQ is to minimize ISI. This issue has been addressed [22] by modifying the SNR definition to include an ISI term:

However, this modified definition is only used to evaluate the performance of the MGSNR TEQ method, which is still based on the de_nition given in (5).

In summary, the drawbacks of the MGSNR TEQ method are that

- its derivation is based on a subchannel SNR de_nitionSNR ${ }^{\mathrm{EQ}}$ that does not include the effect of ISI;

- it depends on the parameter $\mathrm{MSE}_{\max }$ which has to be tuned for different channels;

- its objective function assumes that $\mathrm{b}$ and we are independent; and

- It requires a constrained nonlinear optimization solution.

Considerable effort has been spent to overcome the last issue listed above. Farhang-Boroujeny and Ding [14] propose an eigen-approach based sub-optimum solution to overcome the computational complexity of the constrained nonlinear optimization solver. This approach achieves similar performance with lower computational complexity. For some channels, this sub-optimum approach gives better performance, which proves that the MGSNR TEQ method is not optimum. Lashkarian and Kiaei [12] propose a projection onto convex sets method to iteratively solve the constrained nonlinear optimization problem with lower computational load. Chiu, Tsai, Liau and Troulis [13] reformulate the constrained nonlinear optimization method and propose an inverse power method to solve it. This approach also reduces computational complexity and in some cases gives better performance than the MGSNR TEQ method.

\section{Channel Shortening}

The multicarrier system model is shown in Fig. 1, and the notation is summarized in Table 3.1. Each block of bits is divided up into $\mathrm{N}$ bins, and each bin is viewed as a QAM signal that will be modulated by a 
different carrier. An efficient means of implementing the multicarrier modulation in discrete time is to use an inverse fast Fourier transform (IFFT). The IFFT converts each bin (which acts as one of the frequency components) into a time-domain signal. After transmission, the receiver can use an FFT to recover the data within a bit error rate tolerance, provided that equalization has been performed properly.

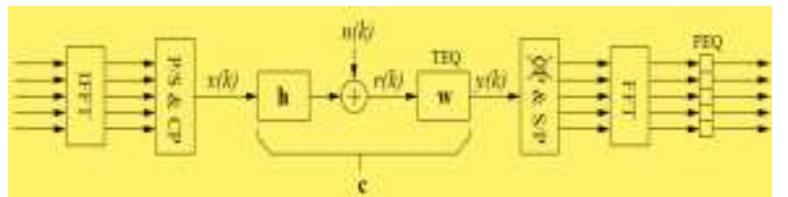

Fig. 1: Traditional multicarrier system model. (I)FFT: (inverse) fast Fourier transform, P/S: parallel to serial, S/P: serial to parallel, CP: add cyclic prefix, and XCP: remove cyclic prefix.

In order for the subcarriers to be independent, the convolution of the signal and the channel must be a circular convolution. It is actually a linear convolution, so it is made to appear circular by adding a cyclic prefix to the start of each data block. The cyclic prefix is obtained by prepending the last $\boldsymbol{v}$ samples of each block to the beginning of the block. If the $\mathrm{CP}$ is at least as long as the channel, then the output of each sub-channel is equal to the input times a scalar complex gain factor. The signals in the bins can then be equalized by a bank of complex gains, referred to as a frequency domain equalizer (FEQ) [18].

The above discussion assumes that $\mathrm{CP}$ length +1 is greater than or equal to the channel length. However, transmitting the cyclic prefix wastes time slots that could be used to transmit data. Thus, the CP is usually set to a reasonably small value, and a TEQ is employed to shorten the channel to this length. In ADSL and VDSL, the $\mathrm{CP}$ length is $1 / 16$ of the block (symbol) length. As discussed in Section I, TEQ design methods have been well explored [2], [3], [4], [5], [6], [7], [8], [9], [10], [11], [12].

One of the TEQ's main burdens, in terms of computational complexity, is due to the parameter, which is the desired delay of the effective channel. The performance of most TEQ designs does not vary smoothly with delay [19], hence a global search over delay is required in order to compute an optimal design. Since the effective channel has Lc +1 taps, there are Lc $+1-v$ locations in which one can place length $v+1$ window of non-zero taps, hence $0 \leq \Delta \leq \mathrm{L}_{\mathrm{c}}-\boldsymbol{v}$. For typical downstream ADSL parameters, this means there are about 500 delay values to examine, and an optimal solution must be computed for each one. One of the goals of this paper is to show how to reuse computations from one value of $\Delta$ to the next, greatly reducing this computational burden.

Inverse-filtering of room acoustics is important for applications such as speech de-reverberation and listening room compensation. Acoustic impulse responses (AIRs) generally have a non-minimum phase characteristic such that a stable single-channel inverse does not necessarily exist. Single-channel approximations to the inverse problem can be obtained by the method of least squares (LS) but they are of limited use in acoustic channel equalization [1]. When multichannel observations are available, the multiple-input/output inverse theorem (MINT) can provide an exact inverse provided the AIRs are known exactly and do not share any common zeros [2].

In the presence of common zeros, MINT and its multichannel least squares (MCLS) formulation [3] can be shown to completely invert those factors that are not common and perform a LS inversion of those parts with common zeros [4]. Subband and iterative algorithms have also been proposed to reduce computational complexity [5,6], although the convergence rate of iterative algorithms is often limited due to ill-conditioning of the problem.

Channel shortening (CS) techniques have been extensively developed in the context of digital communications to mitigate inter-symbol and inter-carrier interference, whereby low-order taps are unconstrained in a so-called relaxation window. A common framework for CS can be found in [7]. In the equalization of acoustic systems, $\mathrm{CS}$ is used to remove audible echoes such that inaudible ones remain, thereby relaxing the design constraints on an equalization filter [8,9]. A generalization of CS as the shortening/reshaping of an AIR with p-norm optimization aims to exploit additional psychoacoustic effects in the design of the equalization filter [10].

Existing inverse-filtering techniques perform well providing the AIR is time-invariant and estimated exactly. However, in many practical scenarios, the AIRs can be disturbed by factors such as source position and temperature that lead to the inversion of a poor estimate of the system [11]. The inverse filter energy is often large, where filter energy is defined as the $` 2$ norm of the filter coefficients. This amplifies small fluctuations in the AIR, leading to increased reverberation rather than a reduction. Efforts to reduce sensitivity to error have involved constraining the filter energy at the expense of reduced de-reverberation [3,1]. Filter gain can also be 
reduced by introducing a modelling delay into the equalization filter so as to relax causality constraints. Previous studies have also considered the effect of noise from the recording apparatus and acoustic sources upon inverse-filtering. It has been shown that constraints placed upon the gain of the equalization filter can improve noise robustness in addition to channel estimation error [3,1]. Channel shortening algorithms have been shown to possess an additional desirable property in that they tend to be more robust to channel estimation errors than existing least-squares techniques [12].

Consider a speech signal recorded in a noisy, reverberant environment with an array of microphones. The observed signals at microphone $m \in\{1,2, \ldots, \mathrm{M}\}$ are given by

$$
\mathbf{x}_{m}(n)=\dot{H}_{m} \mathbf{s}(n)+\nu_{m}(n)
$$

where $\mathrm{s}(\mathrm{n})=[\mathrm{s}(\mathrm{n}) \mathrm{s}(\mathrm{n}-1) \ldots \mathrm{s}(\mathrm{n}-2 \mathrm{~L}+1)] \mathrm{T}, \mathrm{x}_{\mathrm{m}}(\mathrm{n})=\left[\mathrm{x}_{\mathrm{m}}(\mathrm{n}) \mathrm{x}_{\mathrm{m}}(\mathrm{n}-1) \ldots \mathrm{x}_{\mathrm{m}}(\mathrm{n}-\mathrm{L}+1)\right] \mathrm{T}, v_{\mathrm{m}}(\mathrm{n})=\left[v_{\mathrm{m}}(\mathrm{n}) v_{\mathrm{m}}(\mathrm{n}-\right.$ 1) ... $\left.v_{\mathrm{m}}(\mathrm{n}-\mathrm{L}+1)\right] \mathrm{T}$ are segments of the speech signal, noisy observation and noise starting at sample $\mathrm{n}$ respectively and $\mathrm{H}_{\mathrm{m}}$ denotes the $\mathrm{L} \rightarrow(2 \mathrm{~L}-1)$ filtering matrix derived from the AIR. Segments are $\mathrm{L}$ samples in length. The filtering matrix

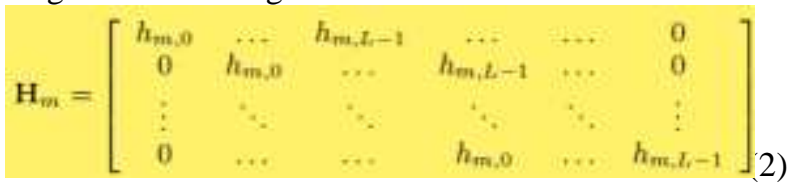

is derived from the filtering vector $h_{m}=\left[h_{m}, 0 \ldots h_{m}, L-1\right] T$. Impulse responses are assumed to be slowly timevarying such that $h_{m}$ is independent of $n$. By concatenating all $M$ outputs of (1), a system of equations

$$
\mathrm{x}(n)=\mathbf{H s}(n)+\nu(n)
$$

can be obtained using the following quantities

$$
\begin{aligned}
& \mathbf{x}(n)=\left[\begin{array}{lll}
\mathbf{x}_{1}^{T}(n) & \mathbf{x}_{2}^{T}(n) \ldots \mathbf{x}_{M}^{T}(n)
\end{array}\right]^{T}, \\
& \mathbf{H}=\left[\begin{array}{llll}
\mathbf{H}_{1}^{T} & \mathbf{H}_{2}^{T} & \ldots & \mathbf{H}_{M}^{T}
\end{array}\right]^{T}, \\
& \nu(n)=\left[\begin{array}{llll}
\nu_{1}^{T}(n) & \nu_{2}^{T}(n) & \ldots & \nu_{M}^{T}(n)
\end{array}\right]^{T} .
\end{aligned}
$$

The noise signals are assumed to be mutually uncorrelated with the source signal. Equalization filters can be calculated by solving the following system of equations

$$
\sum_{m=1}^{M} h_{m}(j) * g_{m}(j)=d(j) \text { for } j=0, \ldots, L+L_{i}-2,
$$

where $\mathrm{Li}$ is the length of $\mathrm{g}_{\mathrm{m}}$ and

$$
d(j)= \begin{cases}0 & \text { if } 0 \leq j<\tau \\ 1 & \text { if } j=\tau \\ 0 & \text { otherwise }\end{cases}
$$

represents the target response with delay $\tau$. In matrix form, (7) can be written as

$$
\tilde{\mathbf{H}} \mathbf{g}=\mathbf{d} \text {, }
$$

where $\mathrm{d}=\left[\mathrm{d}(0) \cdots \mathrm{d}\left(\mathrm{L}+\mathrm{L}_{\mathrm{i}}-2\right)\right] \mathrm{T}$ represents the target responsevector and

$$
\tilde{\mathbf{H}}=\left[\begin{array}{lll}
\tilde{\mathbf{H}}_{1} & \cdots & \tilde{\mathbf{H}}_{M}
\end{array}\right],
$$

with ${ }^{\sim} H_{m}$ an $\left(L+L_{i}-1\right) \rightarrow L_{i}$ filtering matrix of $h_{m}$, 


$$
\tilde{\mathbf{H}}_{m}=\left[\begin{array}{cccc}
h_{m, 0} & 0 & \cdots & 0 \\
h_{m, 1} & h_{m, 0} & \cdots & 0 \\
\vdots & \ddots & \ddots & \vdots \\
h_{m, L-1} & \cdots & \vdots & \vdots \\
0 & h_{m, L-1} & \ddots & \vdots \\
\vdots & \vdots & \ddots & \vdots \\
0 & \ldots & 0 & h_{m, L-1}
\end{array}\right]
$$

Let ${ }^{\wedge} \mathrm{h}$ be an estimate of $\mathrm{h}$ that contains misalignment error, with corresponding inverse filter ${ }^{\mathrm{g}} \mathrm{g}$ and filtering matrix ${ }^{\wedge} \mathrm{G}$ defined in a similar fashion to (5). The aim is to estimate the speech signal s(n) from the noisy, reverberant observations using

$$
\hat{\mathrm{s}}(n)=\hat{\mathbf{G}} \mathrm{x}(n) .
$$

MULTICARRIER modulation (MCM) techniques like orthogonal frequency division multiplexing (OFDM) and discrete multi-tone (DMT) have been gaining in popularity over recent years. One reason for this surge in popularity is the ease with which MCM can combat channel dispersion, provided the channel delay spread is not greater than the length of the cyclic prefix (CP). However, if the cyclic prefix is not long enough, the orthogonality of the sub-carriers is lost and this causes both inter-carrier interference (ICI) and inter-symbol interference (ISI). The inadequacy of the CP in DSL (digital subscriber loop) systems can be seen by considering the standard carrier serving area (CSA) test loops [1].

\section{SYSTEM MODEL}

The channel is divided into bands using the transmitting filters $F_{k}(z)$ and receiving filters $H_{k}(z)$. The input bit stream is parsed and coded as modulation symbols, e.g., PAM or QAM. In [5] and [6], Kalet shows that the DMT system with ideal filters can achieve a signal-to noise-ratio (SNR) within $8-9 \mathrm{~dB}$ of the channel capacity.

In practice, to cancel inter symbol interference (ISI), usually, some degree of redundancy is introduced, and the interpolation ratio $\mathrm{N}>\mathrm{M}$ [1], [2]. The length of the transmitting and receiving filters is usually also N. In the widely used discrete Fourier transform (DFT) based DMT system, the transmitting and receiving filters are DFT filters. Redundancy takes the form of cyclic prefix. For a given probability of error and transmission power, bits can be allocated among the bands to achieve maximum total bit rate $\mathrm{R}_{\mathrm{b} \text {,max }}$. Very high speed data transmission can be achieved using a DFT-based DMT system at a relatively low cost [1]. This technique is currently playing an important role in high speed modems for ADSL and HDSL [3]. Cancelling channel ISI by introducing redundancy using a multi-rate pre-coding technique has been studied by Xia in [7].

In the DMT system, the maximum bit rate $\mathrm{R}_{\mathrm{b} \text {,max }}$ depends on the choice of the transmitting and receiving filters. The use of more general orthogonal transmitting filters instead of DFT filters is proposed in [8]. From the viewpoint of multidimensional signal constellations, it is shown that for additive white Gaussian noise (AWGN) frequency-selective channels, the optimal transmitting and receiving filters are eigenvectors associated with the channel. However, in HDSL applications, the dominating noise is often colored noise known as near end cross talk (NEXT) [1].

We will use the polyphase approach that has enjoyed great success in filter bank theory to study the DMT system [2], [9]. We will derive a modified DFT-based DMT system that has a better noise rejection property but the same cost as the traditional DFT-based system. Moreover, optimal transceiver for colored noise will be studied in detail. In particular, we will show how to assign bits among the bands so that the total transmitting power can be minimized for a given bit rate. Based on the optimal bit allocation, the optimal transceiver is derived. In [6], the DFT-based DMT system is proposed as a practical DMT implementation, but its optimality has not been discussed. We will show that the DFT-based DMT systems are asymptotically optimal, although they are not optimal for a finite number of bands. Furthermore, the asymptotical performance of the DFT-based DMT system is the same as that of the DMT system with ideal filters in [5], [6]. Although the DFT-based DMT system is asymptotically optimal, the optimal transceiver provides significant gain over the DFT-based system for a modest number of bands. 


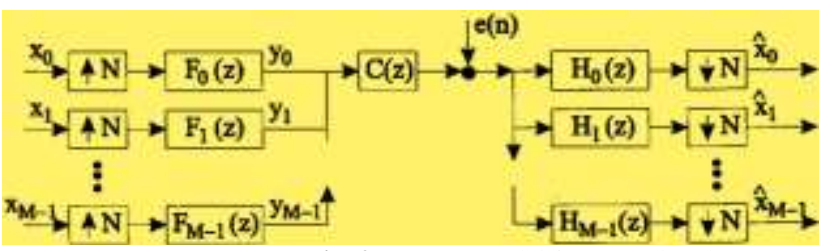

Fig. 2: System model for an M-band DMT system over a frequency-selective channel.

Consider Fig. 1, where an M-band DMT system is shown. Usually, the channel is modelled as an LTI filter $C(z)$ with additive noise $e(n)$. Assume that $C(z)$ is an FIR filter of order L (a reasonable assumption after time domain equalization) and $e(n)$ is a zero-mean wide sense stationary random process. For a given number of bands $M$, the interpolation ratio $N$ is chosen as $N=M+L$. Redundancy is introduced so that the receiver can remove ISI due to $C(z)$ and decoding can be performed block wise. As the interpolation ratio $\mathrm{N} \geq \mathrm{M}$, we say that the system is over interpolated. The filters $F_{k}(z)$ and $H_{k}(z)$ are called transmitting and receiving filters, respectively. In the DMT system, $F_{k}(z)$ and $H_{k}(z)$ have length $=\mathrm{N}$. When the outputs $\wedge x_{k}, k=0,1, \ldots \ldots, M-1$ are identical to the inputs $x_{k}, k=0,1, \ldots \ldots, M-1$, in the absence of channel noise, we say that the system is ISI-free or perfect reconstruction (PR).

The transmitting filters $F_{k}(z)$ have $\mathrm{N}$ coefficients

$$
F_{k}(z)=\sum_{n=0}^{N-1} f_{k}(n) z^{-n}
$$

We can write the $1 \times M$ transmitting bank as

$$
\begin{aligned}
& \left(\begin{array}{llll}
F_{0}(z) & F_{1}(z) & \cdots & F_{M-1}(z)
\end{array}\right) \\
& =\left(\begin{array}{llll}
1 & z^{-1} & \cdots & z^{-(N-1)}
\end{array}\right) \boldsymbol{G}
\end{aligned}
$$

where the $N$ x $M$ matrix G has $[G]_{n, k}=f_{k}(n)$. The implementation of the transmitter is as shown in Fig. 2. Let the receiving filters be Non-causal filters are used here for notational convenience; delays can be added to obtain causal filters. In a similar manner, we can write the receiving bank as

$$
\left(\begin{array}{c}
H_{0}(z) \\
H_{1}(z) \\
\vdots \\
H_{M-1}(z)
\end{array}\right)=S\left(\begin{array}{c}
1 \\
z \\
\vdots \\
z^{N-1}
\end{array}\right)
$$

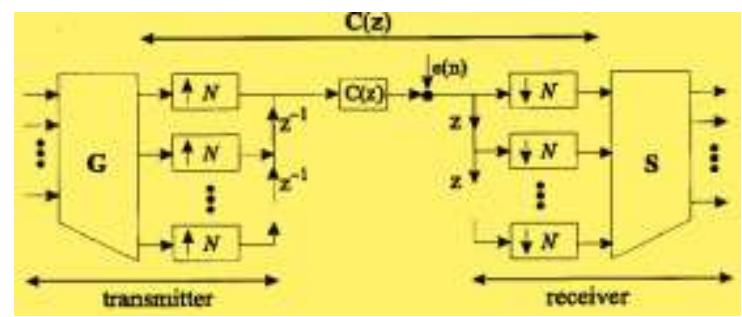

Fig.3: Polyphase representation of the transmitter and the receiver.

where the $N$ x $M$ matrix has $\mathrm{S}$ has $[S]_{n, k}=h_{k}(n)$. The implementation of the receiver is as shown in Fig. 4.2. Using polyphase representation, we can decompose the channel as

$C(z)=\left(\begin{array}{cccc}C_{0}(z) & z^{-1} C_{N-1}(z) & \cdots & z^{-1} C_{1}(z) \\ C_{1}(z) & C_{0}(z) & & \\ \vdots & & \ddots & \vdots \\ C_{N-1}(z) & C_{N-2}(z) & \cdots & C_{0}(z)\end{array}\right)$

Matrices in the above form are known as pseudo-circulant matrices, and their properties can be found in [9]. With the assumption that the channel $C(z)$ is an FIR filter of order, we can write $C(z)=c_{0}+c_{1} z_{-l}+\ldots .+c_{L} z_{-L}$. The channel

Matrix $C(z)$ is pseudo-circulant with the first column given by $\left(\begin{array}{lllllll}c_{0} & c_{1} & \ldots & c_{L} & 0 & \ldots & 0\end{array}\right)^{\mathrm{T}}$. It can be partitioned as a constant matrix and a transfer matrix with $z$ 


$$
C_{0}=\left(\begin{array}{cccccc}
c_{0} & 0 & \cdots & 0 & & 0 \\
c_{1} & c_{0} & & \vdots & & 0 \\
\vdots & & \ddots & & & \vdots \\
c_{L} & c_{L-1} & & & & 0 \\
0 & c_{L} & & & & 0 \\
\vdots & \vdots & \ddots & & & \vdots \\
0 & 0 & & c_{L} & \cdots & c_{0} \\
\vdots & \vdots & & & \ddots & \vdots \\
0 & 0 & & 0 & \cdots & c_{L}
\end{array}\right)
$$

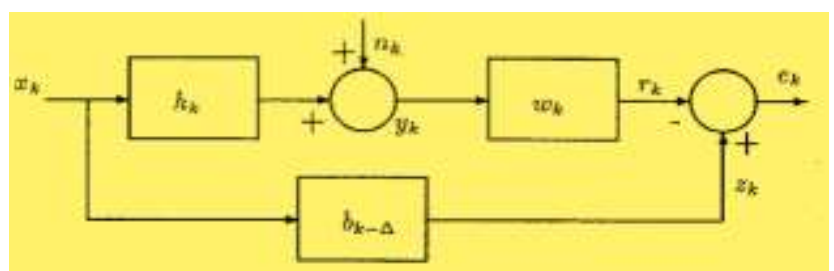

Fig. 4 : Block diagram of FIR - MMSE - IAE.

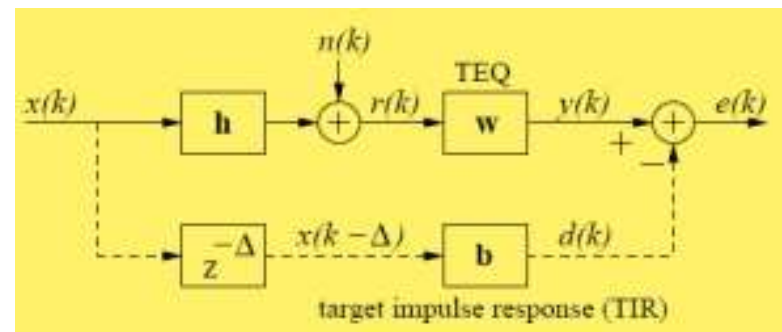

Fig. 5: MMSE system model: $\mathrm{h}, \mathrm{w}$, and $\mathrm{b}$ are the impulse responses of the channel, TEQ, and target, respectively. Here, $\Delta$ represents transmission delay. The dashed lines indicate a virtual path, which is used only for analysis.

The system model for the minimum mean-squared error (MMSE) solution [3] is shown in Fig. 4.4. It creates a virtual target impulse response (TIR) b of length $v+1$ such that the MSE, which is measured between the output of the effective channel and the output of the TIR, is minimized. In the absence of noise, if the input signal is white, then the optimal MMSE and MSSNR solutions are identical [6]. A unified treatment of the MSSNR and noisy MMSE solutions was given in [15].

The MMSE design uses a target impulse response (TIR) b that must satisfy [2]

$$
\mathbf{R}_{r x} \mathbf{b}=\mathbf{R}_{r} \mathbf{w}
$$

Where

$$
\mathbf{R}_{r x}=\mathbf{E}\left[\left[\begin{array}{c}
r(k) \\
\vdots \\
r\left(k-L_{w}\right)
\end{array}\right]\left[\begin{array}{lll}
x(k-\Delta) & \cdots & x(k-\Delta-\nu)]
\end{array}\right]\right.
$$

is the channel input-output cross-correlation matrix and

$$
\mathbf{R}_{r}=\mathbf{E}\left[\left[\begin{array}{c}
r(k) \\
\vdots \\
r\left(k-L_{w}\right)
\end{array}\right]\left[\begin{array}{lll}
r(k) & \cdots & r\left(k-L_{w}\right)
\end{array}\right]\right]
$$

is the channel output autocorrelation matrix. Typically, b is computed first, and then (8) is used to determine w. The goal is that $\mathrm{h} * \mathrm{w}$ approximates a delayed version of $\mathrm{b}$. The target impulse response is the eigenvector corresponding to the minimum eigenvalue of [3], [4], [7]

$$
\mathbf{R}(\Delta)=\mathbf{R}_{x}-\mathbf{R}_{x r} \mathbf{R}_{r}^{-1} \mathbf{R}_{r x} .
$$

Further addresses how to determine most of $\mathrm{R}(\Delta+1)$ from $\mathrm{R}(\Delta)$, and how to use the solution for $\mathrm{b}(\Delta)$ to initialize the eigen solver for $\mathrm{b}(\Delta+1)$. 
There is a tremendous amount of redundancy involved in the brute force calculation of the MSSNR design. This has been addressed in [8]. This section discusses methods of reusing even more of the computations to dramatically decrease the required complexity. Specifically, for a given delay $\Delta$,

- $\mathrm{A}(\Delta)$ can be computed from $\mathrm{B}(\Delta)$ almost for free.

- $\mathrm{B}(\Delta+1)$ can be computed from $\mathrm{B}(\Delta)$ almost for free.

- A shifted version of the optimal MSSNR TEQ w $(\Delta)$ can be used to initialize the generalized eigenvector solution for $\mathrm{w}(\Delta+1)$ to decrease the number of iterations needed for the eigenvector computation.

- $\mathrm{R}(\Delta+1)$ can be computed from $\mathrm{R}(\Delta)$ almost for free.

- A shifted version of the optimal MMSE TIR b $(\Delta)$ can be used to initialize the generalized eigenvector solution for $\mathrm{b}(\Delta+1)$ to decrease the number of iterations needed for the eigenvector computation.

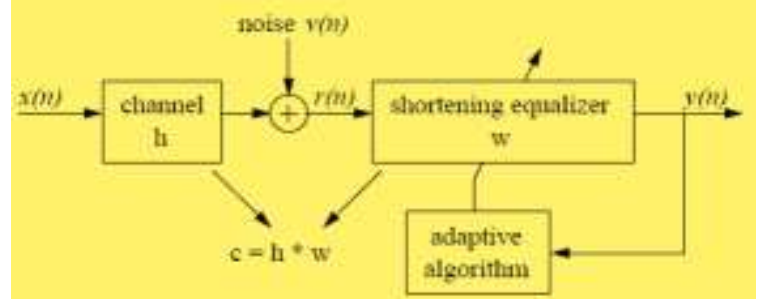

Fig. 5: System model for an adaptive TEQ.

The system model for an adaptive TEQ is shown in Fig. 1. Let $\mathrm{x}(\mathrm{n})$ be the source sequence to be transmitted through a linear finite-impulse-response (FIR) channel h of length $\left(\mathrm{L}_{\mathrm{h}}+1\right)$ taps. Let $\mathrm{r}(\mathrm{n})$ be the received signal, which will be filtered through an $\left(\mathrm{L}_{\mathrm{w}}+1\right)$-tap TEQ with an impulse response vector $\mathrm{w}$ to obtain the output sequence $y(n)$. Let $c=h * w$ denote the effective channel-equalizer impulse response vector of length $\left(L_{c}+1\right)$ taps, where $L_{c}=L_{h}+L_{w}$. The TEQ will be adapted with the goal of shortening the effective channel $c$ such that it possesses significant coefficients only within a contiguous window of size $(v+1)$ taps. In multicarrier systems, $v$ is the CP length. That is, we wish to minimize the energy of the coefficients in the effective channel outside the window of interest. The received sequence $r(n)$ is

$$
r(n)=\sum_{k=0}^{L_{h}} h(k) x(n-k)+v(n),
$$

and the output of the TEQ is

$y(n)=\sum_{k=0}^{L_{w}} w(k) r(n-k)=\mathbf{w}^{T} \mathbf{r}_{n}$,

wherer $_{n}=\left[r(n) r(n-1) \ldots r\left(n-L_{w}\right)\right] T$. Throughout, we make the following assumptions.

1. The source sequence $\mathrm{x}(\mathrm{n})$ is white, zero-mean and wide- sense stationary (W.S.S).

2. The relation $2 \mathrm{Lc}<\mathrm{Nfft}$ holds for multicarrier (or block-based1) systems, i.e. the combined channel has length less than half the FFT (or block) size.

3. The source sequence $x(n)$ is real and has a unit variance.

4. The noise sequence $\mathrm{v}(\mathrm{n})$ is zero-mean, i.i.d., uncorrelated to the source sequence and has a variance $\sigma_{v}{ }^{2}$.

The first assumption is critical for the proposed channel shortening algorithm. Assumption two is important for analytical reasons, but if it is modestly violated the performance degradation should be minor. This assumption is irrelevant for the application of SAM to equalization of (non-CP-based) single carrier systems. The last two assumptions are for notational simplicity. 


\section{OUTPUTS}

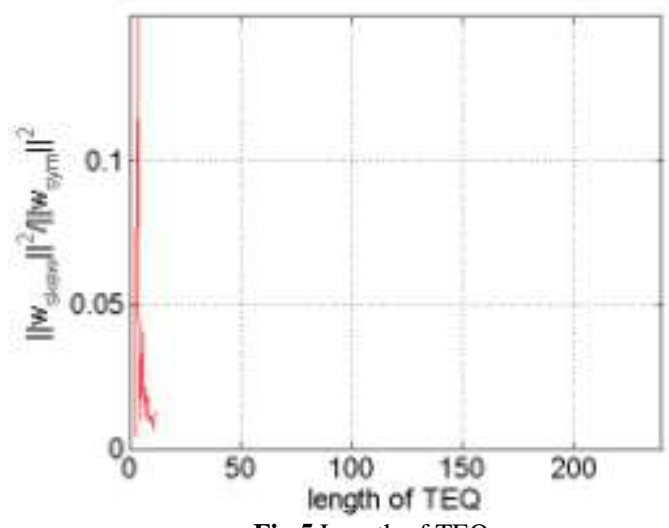

Fig 5 Length of TEQ

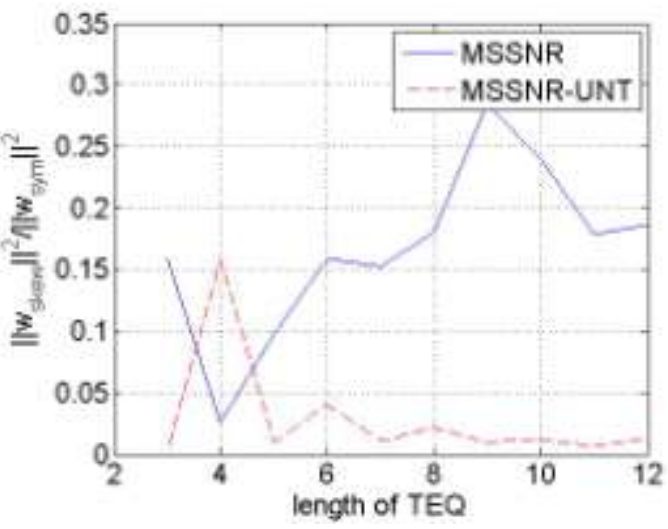

Fig 6 Length of TEQ

Channel-Equalizer Combinations
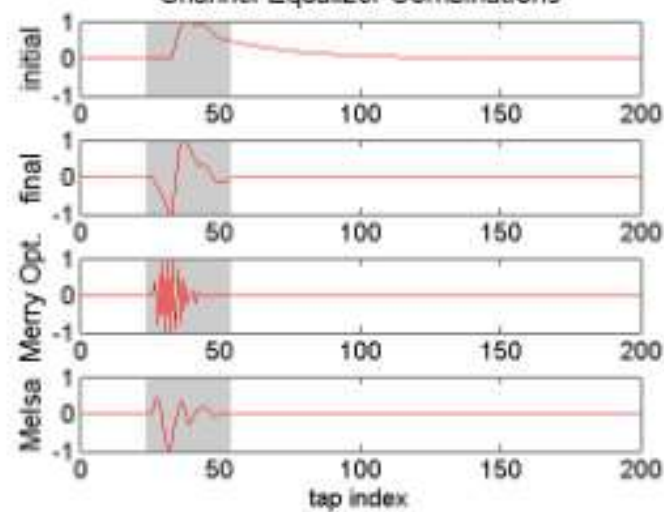

Fig 7 Channel Equilizer Combinations
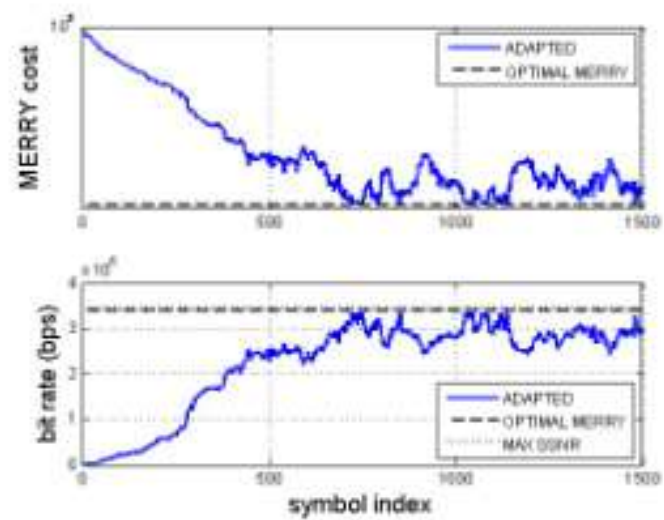

Fig 8 Bitrate VS Symbol Index 


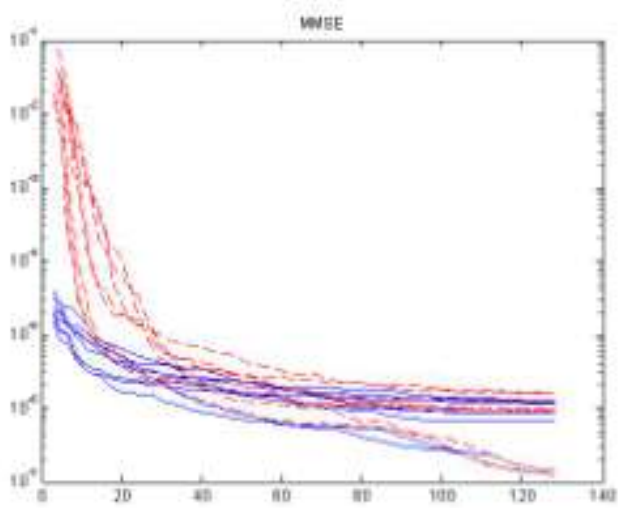

Fig 9 MMSE
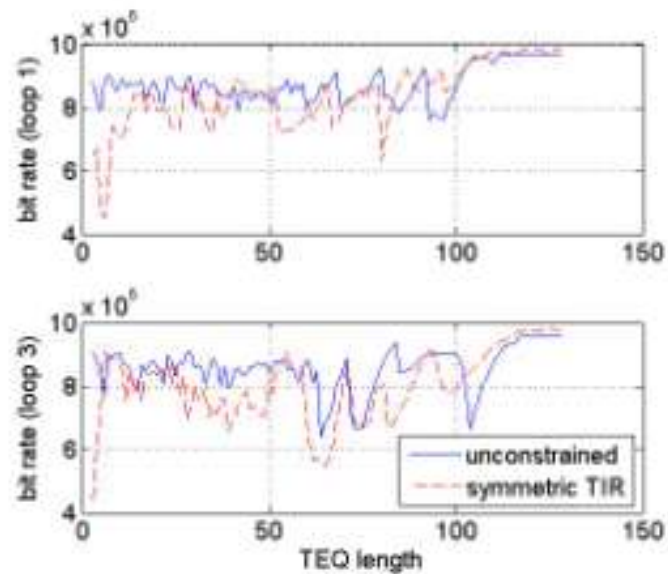

Fig 10 Length of TEQ

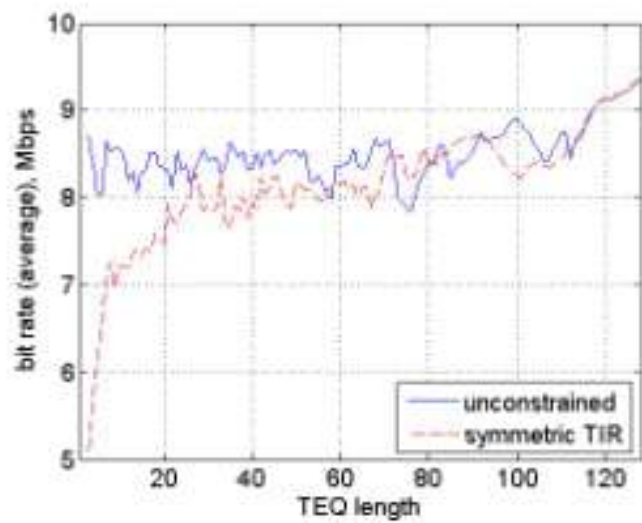

Fig 10 Bitrate VS TEQ Length

\section{CONCLUSION}

In simulations, both the MBR and min-ISI methods outperform previously reported MMSE, MSSNR, and MGSNR methods in bit rate. The min-ISI method delivers virtually equal performance to that of the MBR method. A three-tap TEQ designed by either of the two proposed methods outperforms 17-tap equalizers designed by MMSE, MSSNR, and MGNSR TEQ methods.

A similar technique was proposed to reduce the complexity of computing the $\mathrm{R}(\Delta)$ matrix used in the MMSE design by a factor of 16 (for typical ADSL parameters). It was also shown that the infinite length MSSNR TEQ with a unit norm TEQ constraint has a symmetric impulse response. Simulations are done for Sym-MMSE and Sym-MERRY algorithm. TEQs were designed for CSA loops 1-8, and then the bit rates were averaged.

For an ADSL system, 4-QAM signalling is used on all of the subcarriers during training. Thus, the FEQ can be designed for the training phase by only setting its phase response. The magnitude response can be set after the TEQ is designed. The benefit here is that if the FEQ is designed all at once (both magnitude and phase), then a 
division of complex numbers is required for each tone. However, if the phase response is already known, determining the FEQ magnitude only requires a division of real numbers for each tone.

Thus, if a channel estimate is available, the two possible FEQ phase responses could be determined in parallel with the TEQ design. Similarly, if the TIR is symmetric and the TEQ is long enough that the TIR and effective channel are almost identical, then the phase response of the effective channel is known, except for $\beta$. If differential encoding is used, then the value of ${ }_{\beta}$ can arbitrarily be set to either 0 or ${ }_{\beta}$, since a rotation of exactly 180 degrees does not affect the output of a differential detector.

\section{REFERENCES}

[1] J. A. C. Bingham, IMulticarrier modulation for data transmission: An idea whose time has come," IEEE Comm. Magazine, vol. 28, pp. $5\{14$, May 1990.

[2] T. Starr, J. M. Cio_, and P. J. Silverman, Understanding Digital Subscriber Line Technology. Prentice-Hall PTR, 1999.

[3] J. S. Chow and J. M. Cio_, \A cost-e_ective maximum likelihood receiver for multicarrier systems," in Proc. IEEE Int. Conf. Comm., vol. 2, (Chicago, IL), pp. 948\{952, June 1992.

[4] J. S. Chow, J. M. Cio_, and J. A. Bingham, LEqualizer training algorithms for multicarrier modulation systems," in Proc. IEEE Int. Conf. Comm., vol. 2, (Geneva, Switzerland), pp. 761\{765, May 1993.

[5] I. Lee, J. S. Chow, and J. M. Cio_, IPerformance evaluation of a fast computation algorithm for the DMT in high-speed subscriber loop," IEEE J. on Selected Areas in Comm., vol. 13, pp. 1564\{1570, Dec. 1995.

[6] N. Al-Dhahir and J. M. Cio_, IE_ciently computed reduced-parameter input-aided MMSE equalizers for ML detection: A uni_ed approach," IEEE Trans. on Info. Theory, vol. 42, pp. 903 \{915, May 1996.

[7] M. Na_e and A. Gatherer, ITime-domain equalizer training for ADSL," in Proc. IEEE Int. Conf. Comm., vol. 2, (Montreal, Canada), pp. 1085\{1089, June 1997.

[8] N. Lashkarian and S. Kiaei, IFast algorithm for_nite-length MMSE equalizers with application to discrete multitone modulation," in Proc. IEEE Int. Conf. Acoust., Speech, and Signal Processing, vol. 5, (Phoenix, AR), pp. 2753 22756, Mar. 1999.

[9] P. J. W. Melsa, R. C. Younce, and C. E. Rohrs, IImpulse response shortening for discrete multitone transceivers,"IEEE Trans. on Comm., vol. 44, pp. 1662\{1672, Dec. 1996.

[10] M. Webster and R. Roberts, \Adaptive channel truncation for FFT detection in DMT systems - error component partitioning," in Proc. IEEE Asilomar Conf. on Signals, Systems, and Computers, vol. 1, (Pacific Grove, CA), pp. 669\{673, Nov. 1997.

[11] N. Al-Dhahir and J. M. Cio_, IOptimum _nite-length equalization for multicarrier transceivers," IEEE Trans. on Comm., vol. 44, pp. 56\{63, Jan. 1996.

[12] N. Lashkarian and S. Kiaei, IOptimum equalization of multicarrier systems via projection onto convex set," in Proc. IEEE Int. Conf. Comm., vol. 2, (Vancouver, Canada), pp. 968\{972, June 1999.

[13] W. Chiu, W. K. Tsai, T. C. Liau, and M. Troulis, ITime-domain channel equalizer design using the inverse power method," in Proc. IEEE Int. Conf. Comm., vol. 2, (Vancouver, Canada), pp. 973 \{977, June 1999.

[14] B. Farhang-Bouroujeny and M. Ding, IDesign methods for time-domain equalizers in DMT transceivers," IEEE Trans. on Comm., vol. 49, pp. 554\{562, Mar. 2001.

[15] W. Henkel and T. Kessler, "Maximizing the channel capacity of multicarrier transmission by suitable adaptation of the time-domain equalizer," IEEE Trans. on Comm., vol. 48, pp. $2000\{2004$, Dec. 2000.

[16] D. D. Falconer and F. R. Magee, \Adaptive channel memory truncation for maximum likelihood sequence estimation," Bell System Technical J., vol. 52, pp. $1541\{1562$, Nov. 1973.

[17] B. Wang, T. Adal_, Q. Liu, and M. Vlaynic, IGeneralized channel impulse response shortening for discrete multitone transceivers," in Proc. IEEE Asilomar Conf. on Signals, Systems, and Computers, vol. 1, (Paci_cGrove, CA), pp. $276\{280$, Nov. 1999.

[18] K. V. Acker, G. Leus, M. Moonen, O. V. D. Wiel, and T. Pollet, Per tone equalization for DMT-based systems," IEEE Trans. on Comm., vol. 49, pp. 109\{119, Jan. 2001.

[19] G. Arslan, B. L. Evans, and S. Kiaei, IOptimum channel shortening for discrete multitone transceivers," in Proc. IEEE Int. Conf. Acoust., Speech, and Signal Processing, (Istanbul, Turkey), June 2000.

[20] J. Wu, G. Arslan, and B. L. Evans, IE_cient matrix multiplication methods to implement a near-optimum channel shortening method for discrete multitone transceivers," in Proc. IEEE Asilomar Conf. on Signals, Systems, and Computers, (Paci_c Grove, CA), Nov. 2000. 